\title{
Feature-Tracking Strain Derived from Compressed Sensing Cine Cardiovascular Magnetic Resonance Imaging for Myocardial Infarct Detection: A Feasibility Study
}

\author{
Tomoro Morikawa', Yuki Tanabe ${ }^{1}$, Tomoyuki Kido ${ }^{1}$, Ryo Ogawa', Masashi Nakamura1, \\ Sho Akasaka1, Kouki Watanabe², Michaela Schmidt ${ }^{3}$, Osamu Yamaguchi ${ }^{4}$, Teruhito Kido \\ ${ }^{1}$ Department of Radiology, Ehime University Graduate School of Medicine, Ehime, Japan \\ ${ }^{2}$ Department of Cardiology, Saiseikai Matsuyama Hospital, Ehime, Japan \\ ${ }^{3}$ Siemens Healthcare GmbH, Allee am Roethelheimpark, Erlangen, Germany \\ ${ }^{4}$ Department of Cardiology, Pulmonology, Hypertension and Nephrology, Ehime University Graduate School of Medicine, \\ Ehime, Japan \\ Email: ^tomozo0421@gmail.com
}

How to cite this paper: Morikawa, T., Tanabe, Y., Kido, T., Ogawa, R., Nakamura, M., Akasaka, S., Watanabe, K., Schmidt, M. Yamaguchi, O. and Kido, T. (2021) Feature-Tracking Strain Derived from Compressed Sensing Cine Cardiovascular Magnetic Resonance Imaging for Myocardial Infarct Detection: A Feasibility Study. Open Journal of Radiology, 11, 101-114. https://doi.org/10.4236/ojrad.2021.113010

Received: September 1, 2021

Accepted: September 20, 2021

Published: September 23, 2021

Copyright $\odot 2021$ by author(s) and Scientific Research Publishing Inc. This work is licensed under the Creative Commons Attribution International License (CC BY 4.0).

http://creativecommons.org/licenses/by/4.0/

(c) (i) Open Access

\begin{abstract}
Purpose: This study aimed to use gadolinium-enhanced cardiovascular magnetic resonance (LGE-CMR) scanning to examine the clinical feasibility of feature-tracking strain (FT-strain) analysis on compressed sensing (CS) cine cardiovascular magnetic resonance (CMR) imaging for detecting myocardial infarction (MI). Methods: We enrolled 37 patients who underwent conventional cine CMR, CS cine CMR, and LGE-CMR scanning to assess cardiovascular disease. FT-strain analysis was used to assess peak circumferential strain (p-CS) based on an 18-segment model in both cine CMR imaging modalities. Based on LGE-CMR imaging findings, myocardial segments were classified as remote, adjacent, subendocardial infarcted, and transmural infarcted. The diagnostic performance of $\mathrm{p}$-CS for detecting MI was compared between CS cine CMR imaging and conventional cine CMR imaging using the receiver operating characteristic (ROC) curve analysis. Results: A total of 440 remote, 85 adjacent, 76 subendocardial infarcted, and 65 transmural infarcted segments were diagnosed on LGE-CMR imaging. There were significant between-group differences in p-CS on both conventional and CS cine CMR ( $<0.05$ in each) imaging. The sensitivity and specificity of $\mathrm{p}-\mathrm{CS}$ for identifying MI were $85 \%$ and $79 \%$ for conventional cine CMR imaging, and $82 \%$ and $77 \%$ for CS cine CMR imaging, respectively. There was no significant difference between conventional and CS cine CMR imaging in the area under the curve of $\mathrm{p}-\mathrm{CS}(0.89$ vs. $0.87, \mathrm{p}=0.15)$. Conclusion: FT-strain
\end{abstract}


analysis of CS cine CMR imaging may help identify MI; it may be used alongside or instead of conventional CMR imaging.

\section{Keywords}

Cardiovascular Magnetic Resonance, Cardiac Function, Compressed Sensing, Myocardial Infarction, Myocardial Strain

\section{Introduction}

Assessment of cardiac function is important in patients with cardiovascular disease, for which echocardiography is widely used in clinical practice. Left ventricular ejection fraction (LVEF) is a quantitative index of global cardiac function that enables mortality risk prediction and discrimination [1]. Myocardial strain (longitudinal, circumferential, and radial strain) is useful for the assessment of both global and regional cardiac function [2] [3]. It is easily evaluated using two-dimensional speckle-tracking echocardiography (2D-STE), which has a greater prognostic value than LVEF [4] [5]. However, the robustness of myocardial analysis with 2D-STE depends on the skill of the operator. A blind area on echocardiography may prevent the assessment of the entire myocardium. Cardiovascular magnetic resonance (CMR) imaging has been found to have high objectivity, and it allows for the assessment of the whole myocardium.

Feature-tracking strain (FT-strain) analysis is a method of myocardial strain analysis on CMR scans obtained through cine CMR imaging [6] [7]. Lately, compressed sensing (CS) techniques using sparse sampling and iterative reconstruction have reduced the acquisition time of cine CMR images [8]. FT-strain analysis could potentially be used for cine CMR images with CS, but the clinical feasibility of FT-strain analysis of CS cine CMR images remains unknown. This study aimed to compare FT-strain analysis of CS cine CMR images with that of conventional cine CMR images and evaluate its feasibility for detecting myocardial infarction (MI) using late gadolinium-enhanced (LGE) CMR imaging.

\section{Materials and Methods}

This study was a sub-analysis of a dataset obtained in a previous prospective study [8]. It was approved by an ethics review board of Saiseikai Matsuyama Hospital and has therefore been performed in accordance with the ethical standards laid down in the 1964 Declaration of Helsinki and its later amendments. Informed consent was obtained from all patients enrolled in this study. Seventy-four patients who underwent CMR scanning between August 2014 and May 2015 were included in this study. All patients were scheduled for an evaluation of cardiovascular disease; CMR scanning was performed at the discretion of the attending physician. The CMR protocol included conventional cine CMR, CS cine CMR, and LGE-CMR imaging. The exclusion criteria were as follows: 1) non-ischemic cardiovascular disease, 2) data unsuitable for FT-strain analysis 
(due to arrhythmia or poor breath-holding), and 3) poor LGE-CMR image quality.

\subsection{Cardiovascular Magnetic Resonance Imaging Protocol}

A 3T MR scanner (MAGNETOM Skyra; Siemens Healthcare, Erlangen, Germany) with 48 receivers and two parallel transmission channels was used to implement an established protocol [8]. Scout images were acquired to plan cardiac axial views. Using a segmented balanced steady-state free-precession sequence, conventional cine CMR images of the left ventricle (LV) in the short axis direction were acquired with a stack of eight contiguous slices with adequate slice gaps to cover the entire LV. Additionally, LV short-axis CS cine CMR images were acquired using a prototype sequence with sparse incoherent sampling of $\mathrm{k}$-space and non-linear iterative SENSE-type image reconstruction immediately after the conventional cine CMR scans. Temporal resolution, spatial resolution, and slice orientations were identical in both cine protocols. The details of the procedure used for the acquisition and reconstruction of CS cine CMR images have been reported elsewhere [8]. Over 1.5 cardiac cycles of data were acquired during CS cine CMR imaging. LV short-axis LGE-CMR images were acquired 10 min after the injection of gadobutrol $(0.1 \mathrm{mmol} / \mathrm{kg})$ using a $2 \mathrm{D}$ phase-sensitive inversion recovery sequence. The imaging parameters are presented in Table 1.

Table 1. Imaging parameters.

\begin{tabular}{|c|c|c|c|}
\hline & Conventional cine CMR & CS cine CMR & LGE-CMR \\
\hline Sequence type & 2D cine true FISP & $2 \mathrm{D}$ cine true FISP & 2D PSIR \\
\hline ECG mode & Retrospective gating & Prospective triggering & - \\
\hline TE/TR (ms) & $1.4 / 3.2$ & $1.4 / 3.2$ & $1.3 / 597$ \\
\hline $\mathrm{FOV}(\mathrm{mm})$ & $350 \times 350$ & $350 \times 350$ & $350 \times 350$ \\
\hline Image matrix & $208 \times 166$ & $208 \times 166$ & $133 \times 224$ \\
\hline Spatial resolution $(\mathrm{mm})$ & $1.7 \times 1.7$ & $1.7 \times 1.7$ & $1.6 \times 1.6$ \\
\hline Temporal resolution (ms) & 41 & 41 & 199 \\
\hline Slice thickness (mm) & 6 & 6 & 6 \\
\hline Numbers of slices & 8 & 8 & 12 \\
\hline Slice gap (mm) & $3.6-4.8$ & $3.6-4.8$ & 0.9 \\
\hline Flip angle $\left({ }^{\circ}\right)$ & 50 & 50 & 55 \\
\hline Bandwidth (Hz/pixel) & 1145 & 1145 & 744 \\
\hline Cardiac phases & 25 & $19-31$ & - \\
\hline Numbers of breath-holds & 4 & 1 & $2-3$ \\
\hline Acceleration factor & 3 & 12.8 & 2 \\
\hline Number of iterative reconstructions & - & 80 & - \\
\hline
\end{tabular}

CMR, cardiac magnetic resonance; CS, compressed sensing; ECG, electrocardiogram; FOV, field of view; LGE-CMR, late gadolinium-enhanced magnetic resonance; FISP, fast imaging with steady-state free precession; PSIR, phase-sensitive inversion recovery; TE, echo time; TR, repetition time. 


\subsection{Feature-Tracking Strain Analysis}

All FT-strain analyses were performed using designated software (CMR42; Circle Cardiovascular Imaging Inc, Calgary, Canada). Three representative LV short-axial slices at the basal-, mid-, and apical levels were selected from both short-axial cine CMR and LGE-CMR images by an independent observer. Subsequently, a software algorithm semi-automatically segmented the LV myocardium into six equidistant segments according to the 18-segment model [9], and peak circumferential strain (p-CS) values were independently analyzed in both types of cine CMR images. Inter-observer agreement was assessed using images from 10 randomly selected patients (180 myocardial segments) who were examined by two experienced radiologists with 7 and 15 years of experience in CMR imaging analysis, respectively.

\subsection{LGE-CMR Analysis}

Two experienced radiologists with 8 and 10 years of experience in CMR imaging, respectively, who were blinded to all other data were tasked with independently classifying myocardial characteristics into four groups at the segment level [10] [11]; the groups were defined as follows: 1) a segment without an LGE area that was not adjacent to the border or the infarcted segment was termed the remote segment; 2) a segment without an LGE area that was adjacent to the infarcted segment was termed the adjacent segment; 3) a segment with an LGE transmural extent of $\leq 50 \%$ was termed the subendocardial infarcted segment; and 4) a segment with LGE transmural extent of $>50 \%$ was termed the transmural infarcted segment. The subendocardial and transmural infarcted segments were defined as MI segments. Any discrepancies between two observers were resolved by discussion and consensus.

\subsection{Statistical Analysis}

Continuous data were expressed as mean \pm standard deviation or median (first quartile, third quartile), depending on the type of distribution. Inter-observer agreement for LGE-CMR analysis was evaluated using Cohen's $\kappa$. Inter-observer agreement for $\mathrm{p}$-CS analysis was evaluated using intra-class correlation coefficients (ICC). The paired t-test was used to compare scanning time between conventional and CS cine CMR scans. The Wilcoxon signed-rank test was used to compare the p-CS values between conventional and CS cine CMR scans. Correlation and agreement between the $\mathrm{p}$-CS values obtained from conventional CMR and CS cine CMR images were evaluated using linear regression and the Bland-Altman analyses. Differences in p-CS values among remote, adjacent, and infarcted regions were assessed using the Steel-Dwass test. To analyze diagnostic performance by segment, we used generalized estimating equations, assumed values for exchangeable correlation structures, and adjusted for the clustered nature of the data using robust estimators of variance [12]. The cutoff values and area under the curve (AUC) of p-CS on conventional and CS cine CMR images for detecting myocardial infarction (subendocardial and transmural) were de- 
termined using receiver operating characteristic curve (ROC) analysis. The optimal cut-off values were determined using Youden's index. Sensitivity, specificity, and positive and negative predictive values (PPV and NPV, respectively) with $95 \%$ confidence intervals (CI) were calculated using the cut-off values. In all tests, statistical significance was set at $\mathrm{p}<0.05$. Statistical analyses were performed with JMP14 (SAS Institute, Cary, NC, USA).

\section{Results}

Of the 74 patients initially included in this study, 37 were excluded because of 1) non-ischemic cardiovascular disease $(\mathrm{n}=21), 2)$ data unsuitable for FT-strain analysis $(n=9)$, and 3$)$ poor LGE image quality $(n=7)$. Finally, 37 eligible patients were enrolled (Table 2). All patients had a regular sinus rhythm and a mean heart rate of $60.2 \pm 10.3 \mathrm{bpm}$ during both cine CMR scans. The total examination time was $114.0 \pm 7.0 \mathrm{~s}$ for conventional cine CMR scanning and 24.6 $\pm 4.2 \mathrm{~s}$ for CS cine CMR scanning $(\mathrm{p}<0.0001)$.

\subsection{Characteristics of Myocardial Segments on LGE-CMR Imaging}

A total of 666 segments from 37 patients were classified as remote $(n=440)$, adjacent $(n=85)$, subendocardial infarcted $(n=76)$, or transmural infarcted $(n=$ 65). Among them, 141 (21\%) were defined as MI segments. The inter-observer agreement for LGE-CMR imaging was 0.84 , indicating satisfactory reproducibility $(\kappa>0.70)$.

\section{2. p-CS on Conventional and CS Cine CMR Images}

The p-CS values for conventional and CS cine CMR images were $-19.2 \%$ $(-23.5 \%,-14.0 \%)$, and $-19.7 \%(-23.9 \%,-14.1 \%)$, respectively $(\mathrm{p}=0.21)$. The $\mathrm{p}$-CS values obtained from conventional and CS cine CMR images were significantly correlated $(r=0.75, p<0.05$; Figure $1(A))$. The mean difference between

Table 2. Patient characteristics.

\begin{tabular}{cc}
\hline Age (years) & $70.4 \pm 9.3$ \\
Men (\% of total) & $20(54 \%)$ \\
Body mass index $\left(\mathrm{kg} / \mathrm{m}^{2}\right)$ & $24.1 \pm 3.5$ \\
HR (beats $/ \mathrm{min})$ & $60.2 \pm 10.3$ \\
\hline Cardiovascular risk factors & $21(56 \%)$ \\
\hline Hypertension & $18(48 \%)$ \\
Dyslipidemia & $12(32 \%)$ \\
Diabetes mellitus & $9(24 \%)$ \\
Smoking habit & $3(8 \%)$ \\
Family history of CAD & \\
\hline
\end{tabular}

Data are expressed as mean $\pm \mathrm{SD}$, or $\mathrm{N}(\%)$. CAD, coronary artery disease; $\mathrm{HR}$, heart rate; $\mathrm{SD}$, standard deviation. 
the conventional and CS cine CMR images based on the Bland-Altman method was $0.5 \%$ (95\% limits of agreement: $-8.4 \%$ to $9.4 \%$; Figure $1(\mathrm{~B})$ ). The ICC values were 0.89 (95\% CI: 0.85 - 0.92) and 0.85 (95\% CI: 0.80 - 0.89) for conventional and CS cine CMR images, respectively.

$\mathbf{A}$

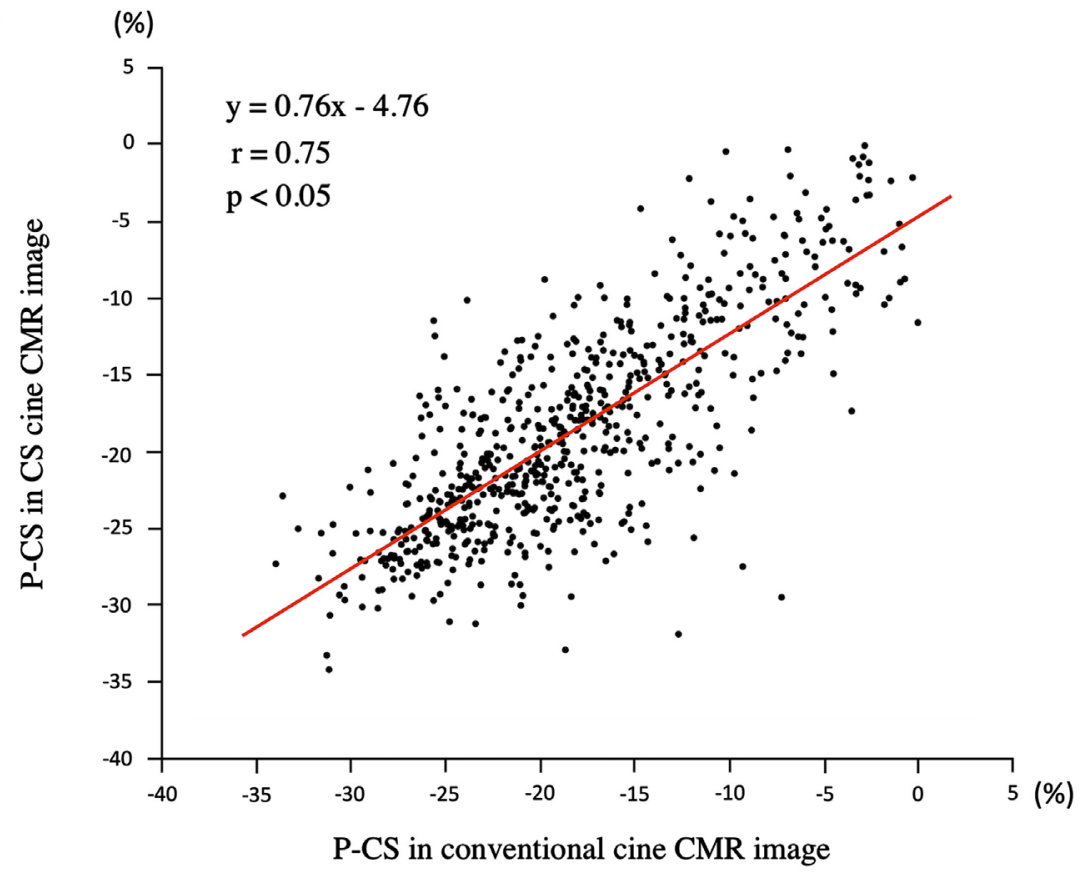

B

(\%)

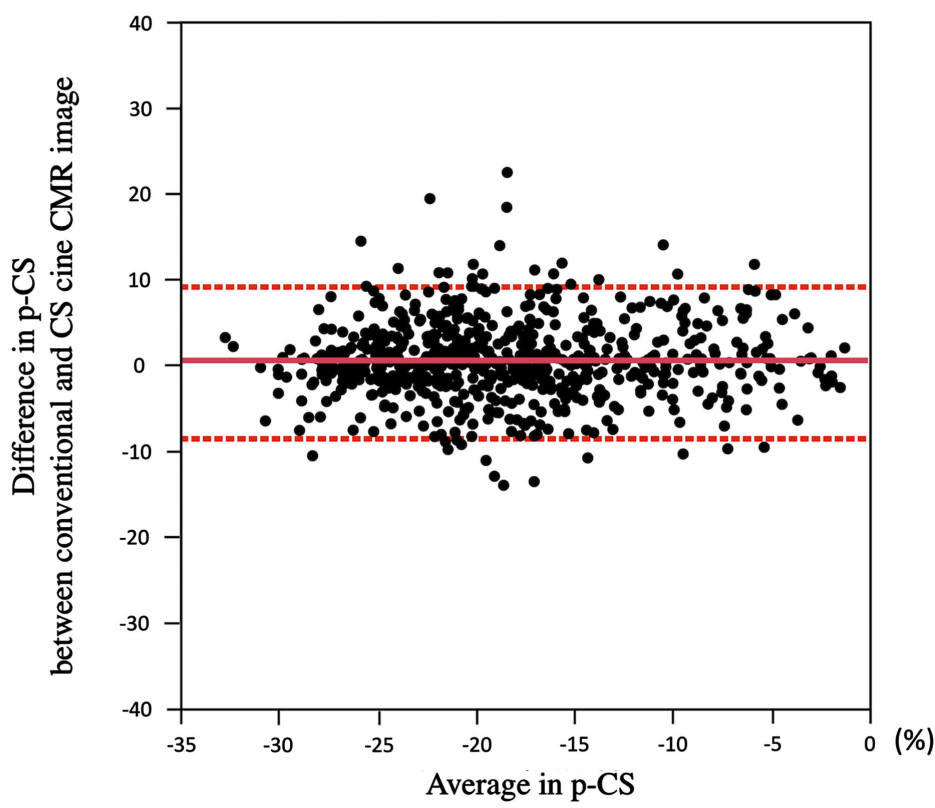

between conventional and CS cine CMR image

Figure 1. Scatter plots (A) and Bland-Altman plots (B) of p-CS on conventional cine CMR and CS cine CMR images. In the Bland-Altman plots, the mean difference between estimates obtained by two techniques is indicated by a solid line; the corresponding double standard deviation intervals are indicated by a dashed line. CMR, cardiac magnetic resonance; CS, compressed sensing; p-CS, peak circumferential strain). 


\subsection{Diagnostic Performance of $p-C S$}

Figure 2 shows the results of p-CS analysis on conventional and CS cine CMR images in a representative case of MI. Figure 3(A) shows the p-CS values in the conventional cine CMR image; they were $-21.6 \%(-24.9 \%,-18.2 \%),-15.3 \%$ $(-19.0 \%,-11.5 \%),-12.3 \%(-15.6 \%,-8.8 \%)$, and $-7.0 \%(-12.2 \%,-3.8 \%)$ for the remote, adjacent, subendocardial infarcted, and transmural infarcted segments, respectively. Figure 3(B) shows the corresponding values in the CS cine CMR image; they were $-22.3 \%(-25.2 \%,-18.4 \%),-15.8 \%(-19.3 \%,-11.6 \%),-13.0 \%$ $(-17.4 \%,-9.4 \%)$, and $-8.8 \%(-12.9 \%,-5.9 \%)$ for the remote, adjacent, subendocardial infarcted, and transmural infarcted segments, respectively. The p-CS values were decreased in all the groups in both conventional and CS cine CMR images (all $\mathrm{p}<0.05$ ).

The diagnostic performance estimates of $\mathrm{p}$-CS for detecting MI (subendocardial and transmural infarcted segments) on conventional and CS cine CMR images are shown in Table 3. ROC curve analysis revealed p-CS AUC values of 0.89 (95\% CI: 0.85 - 0.91) and 0.87 (95\% CI: 0.83 - 0.90) for conventional and CS cine CMR images, respectively ( $\mathrm{p}=0.15$; Figure 4 ). The optimal cutoff value for p-CS was $-16.4 \%$ for conventional CMR images. On the other hand, the optimal cutoff value was $-16.5 \%$ for cine CMR images of CS.

A

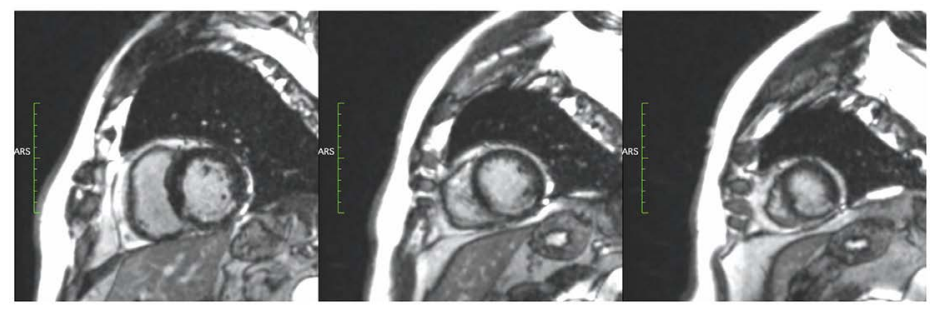

B

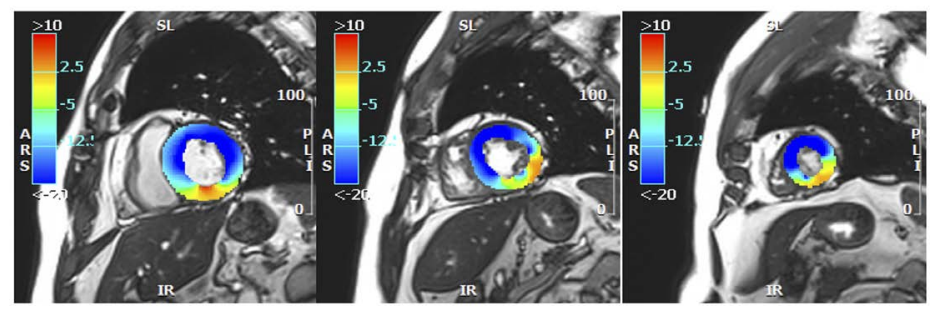

C

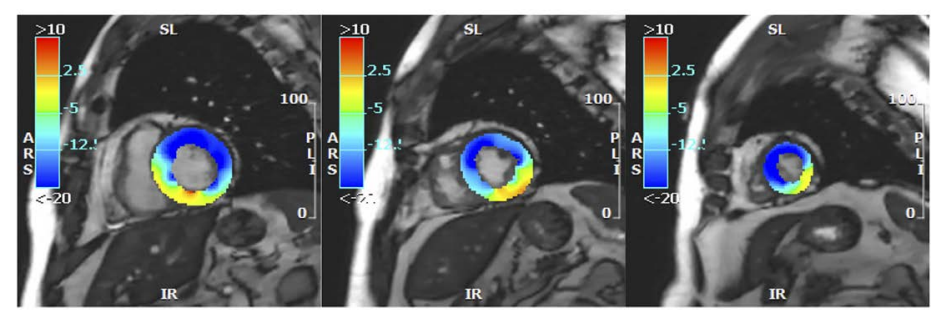

Figure 2. P-CS analysis of conventional cine CMR and CS cine CMR images of a 72-year-old man with myocardial infarction. LGE-CMR shows the late gadolinium enhancement in the LV inferior wall, which is suspected to have undergone infarction (A). The p-CS as assessed using LGE-CMR was decreased in the LV inferior wall on both conventional cine CMR (B) and CS cine CMR (C) images. CMR, cardiac magnetic resonance; CS, compressed sensing; LGE-CMR, late gadolinium-enhanced cardiac magnetic resonance; LV, left ventricle; $p$-CS, peak circumferential strain. 


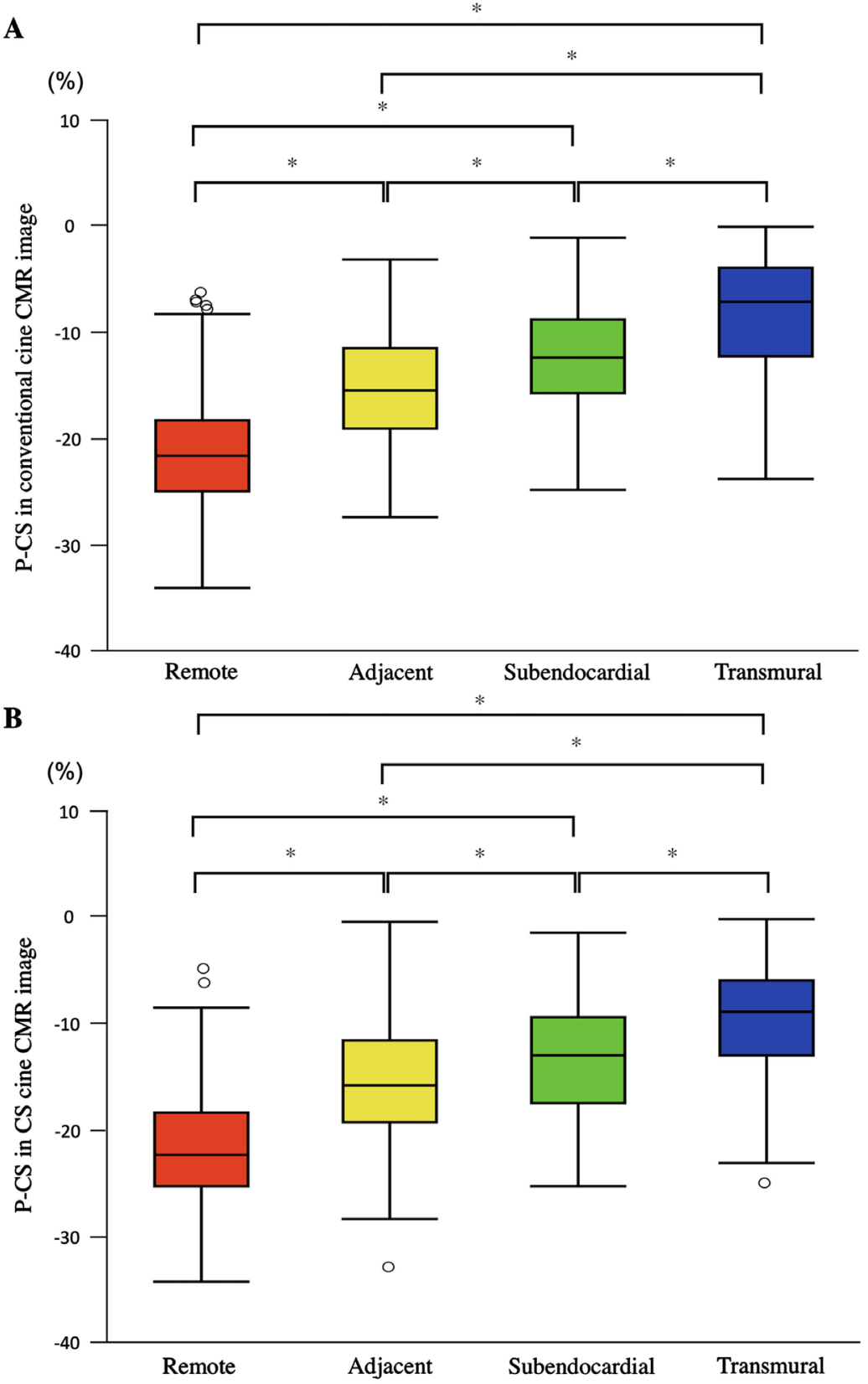

Figure 3. Comparisons of p-CS values among remote, adjacent, subendocardial infarcted, and transmural infarcted segments on conventional cine CMR (A) and CS cine CMR (B) images. CMR, cardiac magnetic resonance; CS, compressed sensing; p-CS, peak circumferential strain. ${ }^{*} \mathrm{p}<0.05$.

Table 3. Diagnostic performance of p-CS on conventional and CS cine CMR images for detecting myocardial infarction.

\begin{tabular}{cccccc}
\hline & $\begin{array}{c}\text { Cut off value } \\
(\%)\end{array}$ & $\begin{array}{c}\text { Sensitivity } \\
(\%)\end{array}$ & $\begin{array}{c}\text { Specificity } \\
(\%)\end{array}$ & PPV (\%) & NPV (\%) \\
\hline $\begin{array}{c}\text { Conventional cine CMR } \\
\text { CS cine CMR }\end{array}$ & $-16.4 \%$ & $85(79-91)$ & $79(76-83)$ & $53(46-59)$ & $95(93-97)$ \\
\hline
\end{tabular}

All values are presented as \% (95\% confidence interval). CMR, cardiac magnetic resonance; CS, compressed sensing; p-CS, peak circumferential strain; NPV, negative predictive value; PPV, positive predictive value. 


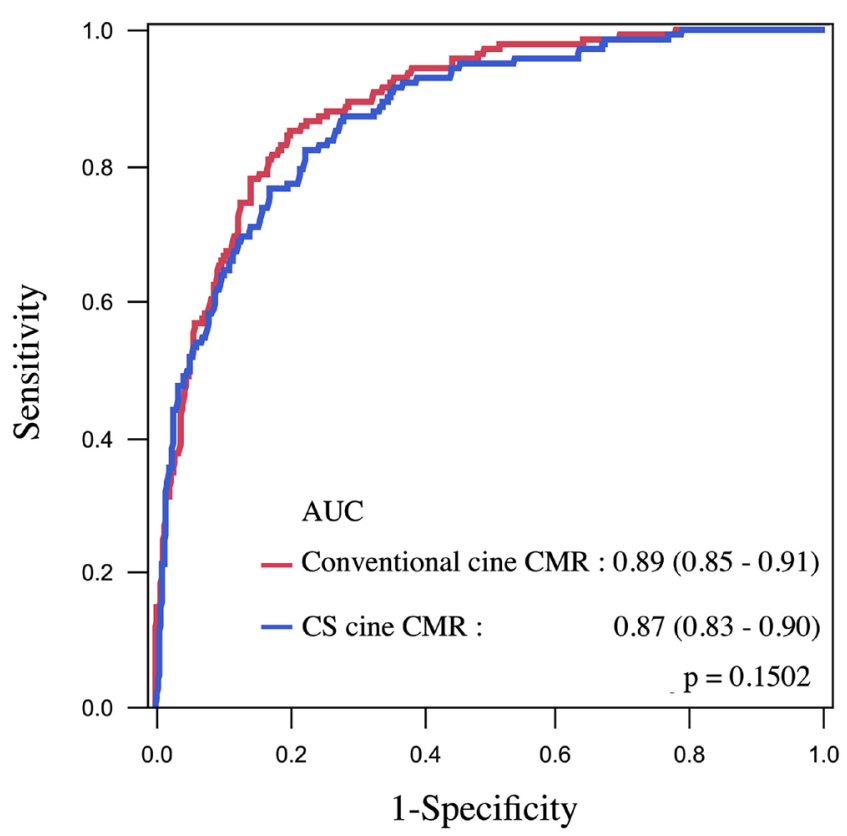

Figure 4. Receiver operating characteristic curve of p-CS for detecting myocardial infarction on conventional cine CMR and CS cine CMR images. CMR, cardiac magnetic resonance; CS, compressed sensing; $\mathrm{p}-\mathrm{CS}$, peak circumferential strain.

\section{Discussion}

The main findings of this study include 1) the significant correlation between the p-CS values of conventional and CS cine CMR images, 2) high diagnostic performance of $\mathrm{p}$-CS for identifying MI segments on conventional and CS cine CMR images, and 3) evidence that p-CS on CS cine CMR imaging may help classify myocardial characterization on LGE-CMR into remote, adjacent, subendocardial infarcted, and transmural infarcted segments.

Myocardial strain analysis on CMR images is a quantitative method of cardiac function assessment. It is conventionally performed using tagging CMR scans or displacement- or strain-encoded imaging [11] [13] [14]. However, additional data must be acquired for strain analysis using these methods. FT-strain analysis can be performed after processing cine CMR images, providing additional diagnostic value to the visual assessment of cardiac function [15]. Recently, the CS technique has been used with cine CMR images, enabling quick data acquisition while maintaining the robustness of cardiac function assessment [8]. In the present study, the p-CS values of CS cine CMR images were correlated with those of conventional cine CMR images. Furthermore, there was no significant difference in p-CS values between conventional and CS cine CMR images, which was previously reported [16]. The present findings indicate that data acquisition of the full cardiac cycle is important for accurate FT-strain analysis using CS cine CMR images, as a prospective real-time cine CMR scan may lack complete end-diastolic phase data, resulting in the underestimation of peak strain values relative to those obtained from retrospective conventional cine CMR scans [16]. 
Consequently, in the present study, we acquired the data of over 1.5 cardiac cycles on CS real-time cine CMR scanning to increase estimate accuracy.

There was no significant difference in the AUC values for detecting MI obtained using conventional and CS cine CMR images. In previous studies, $\mathrm{p}-\mathrm{CS}$ values obtained using conventional CMR strain analysis for detecting MI had a sensitivity estimate of $71 \%-81 \%$ and specificity estimate of $74 \%-75 \%$. These estimates are consistent with the estimated from this study [10] [13]. In the present study, the diagnostic performance of p-CS of CS cine CMR imaging for detecting MI was not inferior to that of conventional cine CMR scanning. Moreover, $\mathrm{p}$-CS values on CS cine CMR images were correlated with the severity and location of MI, as reflected in the categories of remote, adjacent, subendocardial infarcted, and transmural infarcted segments. A previous study has shown that $\mathrm{p}$-CS values obtained from conventional CMR images varied depending on the severity and location of MI on an LGE-CMR image [11]. The use of p-CS on CS cine CMR imaging may help differentiate subendocardial from transmural MI as assessed on LGE-CMR scanning, which is clinically relevant to prognosis and viability after revascularization [17] [18]. Buss et al. have shown that, as with the values obtained from LGE-CMR images, p-CS values from FT-strain analysis may be an independent predictor of cardiac function improvement after revascularization [19].

FT-strain analysis of CS cine CMR images is a quantitative technique of cardiac function assessment that is quicker than conventional CMR strain analysis. CS cine CMR imaging is becoming increasingly available, and FT-strain analysis of CS cine CMR scans may be applied to various cardiovascular diseases, as is presently used on conventional cine CMR scans [20] [21] [22]. This technique, which does not require a contrast medium, may benefit patients who cannot tolerate lengthy examination times and multiple breath-holdings and to those with advanced renal dysfunction and high risk of nephrogenic systemic fibrosis or renal failure.

There are some limitations of the present study. First, this study was conducted at a single center and with a small sample. Second, the present study only used p-CS values, because they are better for detecting MI than other strain parameters (e.g., longitudinal strain) [13] [23]. Further studies using other myocardial strain parameters with CS cine CMR images are required. Third, the reconstruction time for the CS cine CMR image was approximately $3 \mathrm{~min}$. Ongoing technological developments will reduce reconstruction times, thus helping to overcome this limitation.

Fourth, the effects of different types of CMR-FT analysis software on obtained estimates were not assessed. As different software types use different algorithms, they may result in different values; these inherent differences should be considered when comparing findings obtained using different software types [24]. Fifth, the present study did not account for differences in temporal and spatial resolution and regularization during FT strain analysis, the impact of which has been reported [25]. Finally, low temporal resolution or high regularization may 
result in underestimation of strain values [16].

\section{Conclusion}

Myocardial strain is useful for the assessment of both global and regional cardiac function. FT-strain analysis is a method of myocardial strain analysis on CMR scans obtained through cine CMR imaging. CS techniques using sparse sampling and iterative reconstruction can reduce the acquisition time of cine CMR images. In the present study, the FT-strain values of both conventional cine CMR and CS cine CMR decreased in correlation with the degree of myocardial infarction. In conclusion, FT-strain analysis of CS cine CMR images may help identify and classify MI segments; it may be used alongside or instead of that of conventional cine CMR images.

\section{Acknowledgements}

The authors are grateful to Yoshiaki Komori of Siemens Healthcare K.K. (Tokyo, Japan) for the optimization of the sequence parameters and image quality in the present study.

\section{Availability of Data and Material}

The date that supports the findings of this study are available from the corresponding author, T.K., upon reasonable request.

\section{Authors' Contributions}

TM conceived the study, quantitatively measured the strain, performed the statistical analysis, and drafted the manuscript. YT, TK participated in design of the study, assisted in the interpretation of the results, and helped to revise the manuscript. RO, MN quantitatively measured the strain and image contrast. KW participated in the design of the study and its coordination. MS contributed to the sequence development and the implementation on the scanner, as well as the revision of the manuscript. SA, OY and TK assisted with the interpretation of the results and helped revise the manuscript. All authors read and approved the final manuscript.

\section{Ethics Approval}

This study was approved by an ethics review board of Saiseikai Matsuyama Hospital.

\section{Consent to Participate}

Informed consent to participate was obtained from all patients enrolled in this study.

\section{Consent for Publication}

All the participants in this study provided written consent for publication. 


\section{Conflicts of Interest}

The authors declare no conflicts of interest regarding the publication of this paper.

\section{References}

[1] White, H.D., Norris, R.M., Brown, M.A., Brandt, P.W., Whitlock, R.M. and Wild, C.J. (1987) Left Ventricular End-Systolic Volume as the Major Determinant of Survival after Recovery from Myocardial Infarction. Circulation, 76, 44-51. https://doi.org/10.1161/01.CIR.76.1.44

[2] Leitman, M., Lysiansky, M., Lysyansky, P., et al. (2010) Circumferential and Longitudinal Strain in 3 Myocardial Layers in Normal Subjects and in Patients with Regional Left Ventricular Dysfunction. Journal of the American Society of Echocardiography, 23, 64-70. https://doi.org/10.1016/j.echo.2009.10.004

[3] Jeung, M.Y., Germain, P., Croisille, P., El Ghannudi, S., Roy, C. and Gangi, A. (2012) Myocardial Tagging with MR Imaging: Overview of Normal and Pathologic Findings. Radiographics, 32, 1381-1398. https://doi.org/10.1148/rg.325115098

[4] Nahum, J., Bensaid, A., Dussault, C., et al. (2010) Impact of Longitudinal Myocardial Deformation on the Prognosis of Chronic Heart Failure Patients. Circulation: Cardiovascular Imaging, 3, 249-256. https://doi.org/10.1161/CIRCIMAGING.109.910893

[5] Park, J.J., Park, J.B., Park, J.H. and Cho, G.Y. (2018) Global Longitudinal Strain to Predict Mortality in Patients with Acute Heart Failure. Journal of the American College of Cardiology, 71, 1947-1957. https://doi.org/10.1016/j.jacc.2018.02.064

[6] Augustine, D., Lewandowski, A.J., Lazdam, M., et al. (2013) Global and Regional Left Ventricular Myocardial Deformation Measures by Magnetic Resonance Feature Tracking in Healthy Volunteers: Comparison with Tagging and Relevance of Gender. Journal of Cardiovascular Magnetic Resonance, 15, 8. https://doi.org/10.1186/1532-429X-15-8

[7] Khan, J.N., Singh, A., Nazir, S.A., Kanagala, P., Gershlick, A.H. and McCann, G.P. (2015) Comparison of Cardiovascular Magnetic Resonance Feature Tracking and Tagging for the Assessment of Left Ventricular Systolic Strain in Acute Myocardial Infarction. European Journal of Radiology, 84, 840-848. https://doi.org/10.1016/j.ejrad.2015.02.002

[8] Kido, T., Kido, T., Nakamura, M., et al. (2016) Compressed Sensing Real-Time Cine Cardiovascular Magnetic Resonance: Accurate Assessment of Left Ventricular Function in a Single-Breath-Hold. Journal of Cardiovascular Magnetic Resonance, 18, 50. https://doi.org/10.1186/s12968-016-0271-0

[9] Nakai, H., Takeuchi, M., Otani, K., et al. (2008) Assessment of Left Ventricular Dyssynchrony in Myocardial Infarction Using 2D Speckle Tracking Imaging. Journal of Echocardiography, 6, 109-118. https://doi.org/10.2303/jecho.6.109

[10] Ogawa, R., Kido, T., Nakamura, M., et al. (2017) Diagnostic Capability of Feature-Tracking Cardiovascular Magnetic Resonance to Detect Infarcted Segments: A Comparison with Tagged Magnetic Resonance and Wall Thickening Analysis. Clinical Radiology, 72, 828-834. https://doi.org/10.1016/j.crad.2017.05.010

[11] Inoue, Y., Yang, X., Nagao, M., et al. (2010) Peri-Infarct Dysfunction in Post-Myocardial Infarction: Assessment of 3-T Tagged and Late Enhancement MRI. European Radiology, 20, 1139-1148. https://doi.org/10.1007/s00330-009-1657-2

[12] Sternberg, M.R. and Hadgu, A. (2001) A GEE Approach to Estimating Sensitivity 
and Specificity and Coverage Properties of the Confidence Intervals. Statistics in Medicine, 20, 1529-1539. https://doi.org/10.1002/sim.688

[13] Oyama-Manabe, N., Ishimori, N., Sugimori, H., et al. (2011) Identification and Further Differentiation of Subendocardial and Transmural Myocardial Infarction by Fast Strain-Encoded (SENC) Magnetic Resonance Imaging at 3.0 Tesla. European Radiology, 21, 2362-2368. https://doi.org/10.1007/s00330-011-2177-4

[14] Feng, L., Donnino, R., Babb, J., Axel, L. and Kim, D. (2009) Numerical and in Vivo Validation of Fast Cine Displacement-Encoded with Stimulated Echoes (DENSE) MRI for Quantification of Regional Cardiac Function. Magnetic Resonance in Medicine, 62, 682-690. https://doi.org/10.1002/mrm.22045

[15] Scatteia, A., Baritussio, A. and Bucciarelli-Ducci, C. (2017) Strain Imaging Using Cardiac Magnetic Resonance. Heart Failure Reviews, 22, 465-476.

https://doi.org/10.1007/s10741-017-9621-8

[16] Kido, T., Hirai, K., Ogawa, R., et al. (2021) Comparison between Conventional and Compressed Sensing Cine Magnetic Resonance Feature Tracking. Journal of Cardiovascular Magnetic Resonance, 23, 10. https://doi.org/10.1186/s12968-021-00708-5

[17] Kim, R.J., Hillenbrand, H.B. and Judd, R.M. (2000) Evaluation of Myocardial Viability by MRI. Herz, 25, 417-430. https://doi.org/10.1007/s000590050034

[18] Kim, R.J., Wu, E., Rafael, A., et al. (2000) The Use of Contrast-Enhanced Magnetic Resonance Imaging to Identify Reversible Myocardial Dysfunction. The New England Journal of Medicine, 343, 1445-1453. https://doi.org/10.1056/NEJM200011163432003

[19] Buss, S.J., Krautz, B., Hofmann, N., et al. (2015) Prediction of Functional Recovery by Cardiac Magnetic Resonance Feature Tracking Imaging in First Time ST-Elevation Myocardial Infarction. Comparison to Infarct Size and Transmurality by Late Gadolinium Enhancement. International Journal of Cardiology, 183, 162-170. https://doi.org/10.1016/j.ijcard.2015.01.022

[20] Taylor, R.J., Umar, F., Lin, E.L., et al. (2016) Mechanical Effects of Left Ventricular Midwall Fibrosis in Non-Ischemic Cardiomyopathy. Journal of Cardiovascular Magnetic Resonance, 18, 1. https://doi.org/10.1186/s12968-015-0221-2

[21] Amaki, M., Savino, J., Ain, D.L., et al. (2014) Diagnostic Concordance of Echocardiography and Cardiac Magnetic Resonance-Based Tissue Tracking for Differentiating Constrictive Pericarditis from Restrictive Cardiomyopathy. Circulation: Cardiovascular Imaging, 7, 819-827. https://doi.org/10.1161/CIRCIMAGING.114.002103

[22] Taylor, R.J., Umar, F., Panting, J.R., Stegemann, B. and Leyva, F. (2016) Left Ventricular Lead Position, Mechanical Activation, and Myocardial Scar in Relation to Left Ventricular Reverse Remodeling and Clinical Outcomes after Cardiac Resynchronization Therapy: A Feature-Tracking and Contrast-Enhanced Cardiovascular Magnetic Resonance study. Heart Rhythm, 13, 481-489.

https://doi.org/10.1016/j.hrthm.2015.10.024

[23] Al Musa, T., Uddin, A., Swoboda, P.P., et al. (2017) Myocardial Strain and Symptom Severity in Severe Aortic Stenosis: Insights from Cardiovascular Magnetic Resonance. Quantitative Imaging in Medicine and Surgery, 7, 38-47. https://doi.org/10.21037/qims.2017.02.05

[24] Schuster, A., Stahnke, V.C. and Unterberg-Buchwald, C. (2015) Cardiovascular Magnetic Resonance Feature Tracking Assessment of Myocardial Mechanics: Intervendor Agreement and Considerations Regarding Reproducibility. Clinical Radiology, 70, 989-998. https://doi.org/10.1016/j.crad.2015.05.006 
[25] Knobelsdorff-Brenkenhoff, F., Schunke, T., Reiter, S., Scheck, R., Höfling, B. and Pilz, G. (2020) Influence of Contrast Agent and Spatial Resolution on Myocardial Strain Results Using Feature Tracking MRI. European Radiology, 30, 6099-6108. https://doi.org/10.1007/s00330-020-06971-X 